\title{
High range free space optic transmission using new dual diffuser modulation technique
}

\author{
A.K Rahman ${ }^{1}$, N Julai ${ }^{1}, M$ Jusoh $^{2}$,C.B.M.Rashidi ${ }^{2}$, S.A Aljunid ${ }^{2}$, Anuar M.S ${ }^{2}$, M.F Talib ${ }^{2}$, Nurdiani Zamhari ${ }^{1}$, S.K

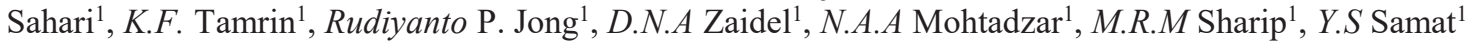

1 Faculty of Engineering, Department of electrical electronic, University Malaysia Sarawak, Malaysia

2 Advance Communication Engineering, Centre of Excellence, SCCE, University Malaysia Perlis, Malaysia

\begin{abstract}
Free space optical communication fsoc is vulnerable with fluctuating atmospheric. This paper focus analyzes the finding of new technique dual diffuser modulation (ddm) to mitigate the atmospheric turbulence effect. The performance of fsoc under the presence of atmospheric turbulence will cause the laser beam keens to (a) beam wander, (b) beam spreading and (c) scintillation. The most deteriorate the fsoc is scintillation where it affected the wavefront cause to fluctuating signal and ultimately receiver can turn into saturate or loss signal. Ddm approach enhances the detecting bit ' 1 ' and bit ' 0 ' and improves the power received to combat with turbulence effect. The performance focus on signal-to-noise (snr) and bit error rate (ber) where the numerical result shows that the ddm technique able to improves the range where estimated approximately $40 \%$ improvement under weak turbulence and $80 \%$ under strong turbulence.
\end{abstract}

\section{Introduction}

Free space optical communication is an attractive alternative over fiber optical communication where provides high bandwidth, fast-installation and high security [1].

However FSOC is suffering with the atmospheric turbulence which can lead the laser beam (a) beam wanders, (b) beam spreading and (c) scintillation. The beam wanders is refer to the condition beam deflected randomly through the changing refractive index oddities. Meanwhile beam spreading refers to conditions where the beam spread more than diffraction estimated predict. Lastly, for scintillation it affected the phase front of the beam can vary and resulting fluctuation irradiance or well known as intensity signal. The combination of all these effect will cause both the spatial and temporal experience random fluctuations in refractive index of the variability of element factor such as temperature, pressure and wind variations along the optical propagation path through the channel [2-9].

In this paper we make the comparison performance dualdetection approach with the conventional approach in term of turbulence strength. In section 2 we outline the model dual-detection approach with assume using OOK modulation. In section 3 we derive the theoretical analysis for SNR and BER. In section 4 results and discussion and section 5 presents the conclusion

\section{Dual Diffuser Modulation Technique}

The system employs two transmitters and On-Off Shift Keying (OOK) modulation as reference for conventional system. When the first transmitter sends binary ' 1 ', the second transmitter which is set in compliment condition will send the binary ' 0 ' in simultaneously and vice versa.

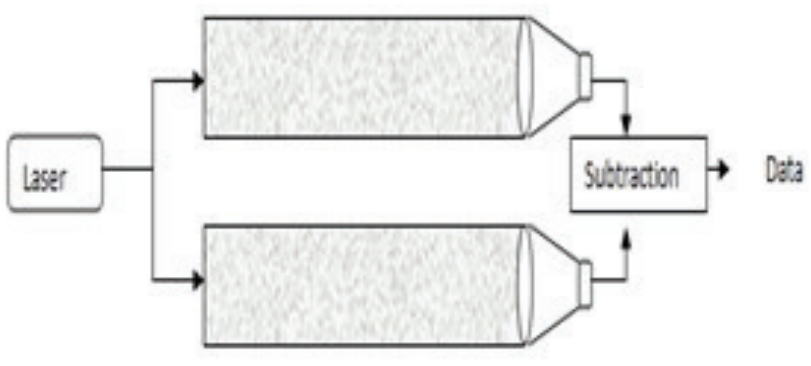

atrosshericturbulence

Fig. 1. Dual Diffuser Modulation technique setup in free space optical communication

Meanwhile at the receiver part, the signal will go through the subtractor for the differential detection process. Here we assume the ideal subtractor condition where no losses signal occurs during subtraction. Therefore, the signal output will become bit ' 1 ' for sending binary ' 1 ' and bit '-1' for sending binary ' 0 '.

This condition leads to modification on conventional OOK modulation particularly in improving signal threshold detection and reduce power loss.

\section{Theory Of Analysis}

Based on the Rytov theory, the field of the plane wave in random media is given [10]:

$$
E_{o}(\vec{r})=A_{o}(\vec{r}) \exp \left(i \varphi_{o}(\vec{r})\right)
$$

Where $A_{o}(\vec{r})$ is amplitude of the laser beam in the atmosphere without the presence of turbulence. $\varphi_{o}$ and $E_{o}(\vec{r})$ are the phase and laser beam profile, respectively. 
However in the presence of turbulence, the scintillation effect is more dominant which change the refractive index and as a consequence lead to intensity fluctuations. Therefore this condition directly change the laser beam profile $[10,11]$ :

$$
E_{o}(\vec{r})=A_{o}(\vec{r}) \exp \left(i \varphi_{o}(\vec{r})\right)=E_{o}(\vec{r}) \exp (\Phi)
$$

Where $A(r)$ is amplitude of the laser in the turbulence atmospheric, $\phi$ is the exponential factor due to turbulence given as:

$$
\begin{aligned}
& \Phi=\operatorname{Ln}\left(\frac{A(\vec{r})}{A_{o}(\vec{r})}\right)+i\left[\phi(\vec{r})-\phi_{o}(\vec{r})\right] \\
& =\chi+i S
\end{aligned}
$$

Where $X$ represents the fluctuation of the log-amplitude of the field and $S$ is the phase fluctuations. This equation predicts a fluctuation in intensity and phase of receiving signal due to turbulence is weak and assuming that the refractive index structure coefficient $C_{n}^{2}$ is symmetrical along the beam path, Rytov suggested a variance for the logirradiance given as [6]:

$\sigma_{\ln I R}^{2}=\left\langle(\ln I-\langle\ln I\rangle)^{2}\right\rangle=1.23 C_{n}^{2} k^{7 / 6} L^{11 / 6}$

Where $k$ is wave number, $k=2 \pi / \lambda, L$ is propagation distance and $I$ is intensity signal. However in practical FSO for plane wave in weak condition and for the symmetrical turbulence of the log-amplitude fluctuation is given by $[9,10]$ :

$$
\left\langle\chi^{2}\right\rangle=0.31 C_{n}^{2} k^{7 / 6} L^{11 / 6}
$$

By neglecting other source noises, we assume the only dominant noise source is atmospheric turbulence which gives the log-amplitude as:

$\chi=\operatorname{Ln}\left(\frac{A(\vec{r})}{A_{o}(\vec{r})}\right)=\ln \left[\frac{A_{o}(\vec{r})+A_{n}(\vec{r})}{A_{o}(\vec{r})}\right]=\ln (1+\varepsilon)$

Where $A_{n}(\vec{r})$ is the amplitude of noise and $\varepsilon=A_{n}(\vec{r}) /$ $A_{o}(\vec{r})$ is the ratio of the amplitude of noise to signal. Both SNR and BER are used to evaluate the quality of the communication systems. The BER performance depends on the average received power, the scintillation strength, receiver noise and threshold bits ( 1 or 0$)$ detection. Here the dual-detection approach is a modification from the conventional OOK detection that using direct detection where improves power received and eliminated the need of complex adaptive threshold. The SNR for case only dominant turbulence noise in term of mean signal and noise intensity $I_{o}$ and $<I_{n}>$ given as:

$S N R=\frac{I_{O}}{\left\langle I_{n}\right\rangle}=\frac{\left\langle A_{O}^{2}(\vec{r})\right\rangle}{\left\langle A_{n}^{2}(\vec{r})\right\rangle}=\frac{1}{\left\langle\varepsilon^{2}\right\rangle}$
So, the BER for OOK modulation can be written as [10]:

$B E R=\frac{1}{2} \operatorname{erfc}\left(\frac{Q}{2}\right)=\frac{e^{\left(-\frac{Q^{2}}{2}\right)}}{Q \sqrt{2 \pi}}$

where $\operatorname{erfc}()$ the complementary error function and $Q$ is defined as:

$Q=\frac{A_{1}-A_{0}}{\sigma_{1}+\sigma_{0}}$

Where $A_{l}, \sigma_{l}$ are the mean signal and noise at the receiver for a bit ' 1 ' respectively, and for a bit ' 0 ' the mean signal and noise are denoted as $A_{0}, \sigma_{0}$.

For conventional OOK bit detection,

$A_{1}=\left\langle A_{o}(\vec{r})\right\rangle, A_{o}=0$ and $\sigma_{1}+\sigma_{0}=\left\langle A_{o}(\vec{r})\right\rangle$

$S N R=\frac{<A_{o}^{2}(\vec{r})>}{<A_{n}^{2}(\vec{r})>}=Q^{2}$

However for case dual-detection approach,

$A_{1}=\left\langle A_{o}(\vec{r})\right\rangle, A_{o}=\left\langle-A_{o}(\vec{r})\right\rangle$ and $\sigma_{1}+\sigma_{0}=\left\langle A_{o}(\vec{r})\right\rangle$

$S N R=\frac{\left.<2 A_{o}^{2}(\vec{r})\right\rangle}{\left.<A_{n}^{2}(\vec{r})\right\rangle}$

For weak turbulence model, $\varepsilon$ is very small thus (6) is given as [10]:

$\chi=\ln (1+\varepsilon) \approx \varepsilon$

When turbulence is strong, the SNR and $\chi$ can be relating using Tailor series for function $f(x)=\left(e^{x}-1\right)^{2}$ and can be approximately to:

$S N R=\frac{1}{\left\langle\chi^{2}+\chi^{3}+\cdots \cdot\right\rangle} \cong \frac{1}{\alpha\left\langle\chi^{2}\right\rangle}$

\section{Results and Discussion}

In this numerical result section, we use parameters wavelength $1550 \mathrm{~nm}, \mathrm{Cn}^{2}=1 \times 10-15$ for weak turbulence and $\mathrm{Cn} 2=1 \times 10-14$ for strong turbulence and range propagation $5 \mathrm{~km}$ for all calculations.

In Figure 2 shows the $\mathrm{SNR}$ in $\mathrm{dB}$ response to turbulence strength. From the graph pattern, we can know that as the distance increase, the SNR also decrease. The SNR is influenced by turbulence strength. Therefore by using the dual-detection approach the SNR can improve approximately $3 \mathrm{~dB}$ or $17 \%$ compare to the conventional approach.

In Figure 3 shows the BER versus range propagation in response to weak turbulence. By using the standard communication for acceptable BER at 10-9, the conventional approach only reaches the range propagation $2.5 \mathrm{~km}$ but the dual-detection approach can reach further to $3.5 \mathrm{~km}$ which approximately $40 \%$ improvement. 


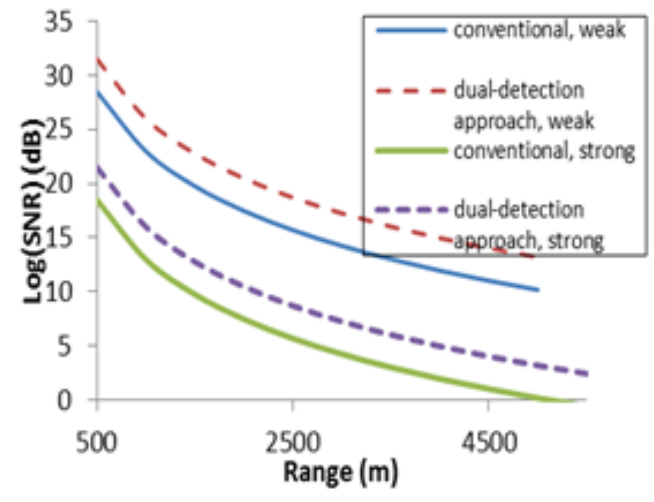

Fig. 2: SNR (dB) versus range propagation

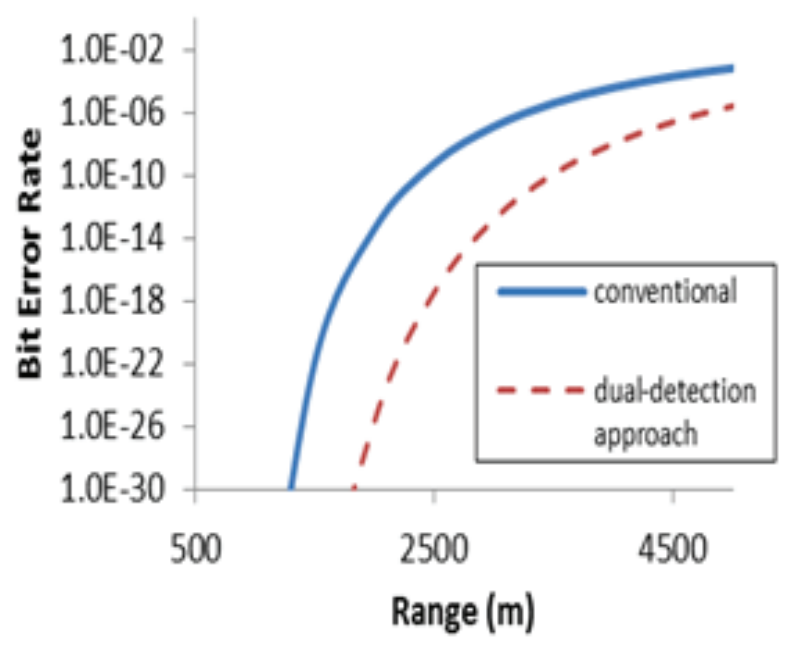

Fig. 3: BER versus range propagation for weak turbulence condition

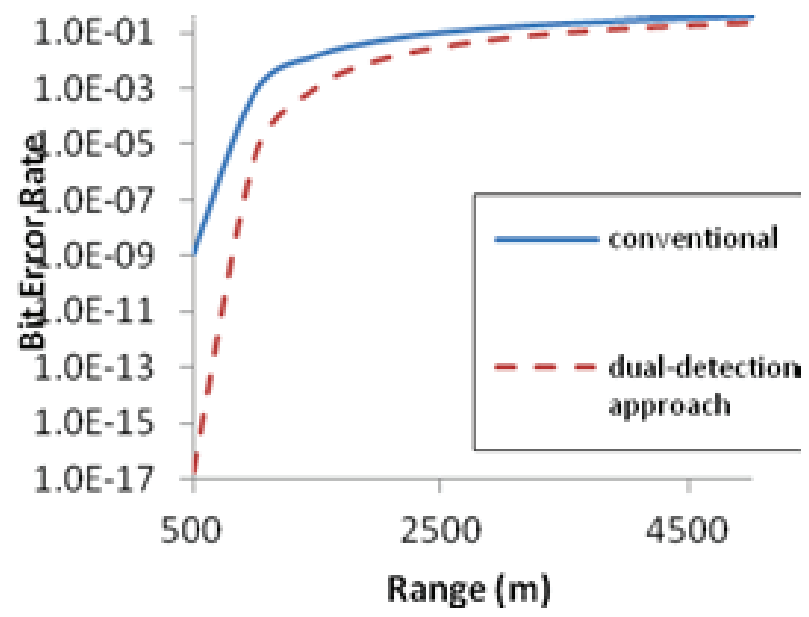

Fig. 4: BER versus range propagation for strong turbulence condition

In Figure 4 shows the BER versus range propagation for strong turbulence condition. Here we assume the value of $\alpha$ is 2 . In range of good quality communication (BER=10$9)$, the conventional approach only reaches the range propagation about $0.5 \mathrm{~km}$ but the dual-detection approach still manage to reach $0.9 \mathrm{~km}$ where about $80 \%$ improvement.

\section{Conclusions}

This paper focuses on the turbulence effect that deteriorates the performance of FSOC using new technique that is DDM. The comparison performance measured in SNR and BER between two approaches that are conventional (OOK-direct detection) and DDM. Analyses that carried out shows that the proposed DDM technique 
improve the margin SNR and BER. The range of propagation can be predicted improved $40 \%$ in weak turbulence and $80 \%$ in strong turbulence.

This research is supported under UNIMAS MyRA - Special Short Term Grant (SpSTG) scheme.

\section{References}

1. Kim, Isaac, Opt. Eng. SPIE, 37, 12, pp. 3143-3155 (1998).

2. N. Namazi, R. Burris, and G. C.Gilbreath, Proc. of SPIE, 5892, pp. (58920T) 1-15, (2005).

3. S. Trisno, I. Smolyaninov, S. D. Milnerb, and C. C. Davis, Proc. of SPIE, 5892, pp. (589215) 1-10, (2005).

4. M. C. R. Cordeiro, C. P. Colvero, and J. P. von der Weid, Int. Conf. on Microwave and Optoelec., pp. 393395, (2005).

5. M. Uysal, J. Li, and M. Yu, IEEE Transaction on Wireless Communications, 5, 6, pp. 1229-1233, (2006).

6. I. B. Djordjevic, B. Vasic, and M. A. Neifeld, IEEE Photon. Techn. Let. 18, 14, pp. 1491-1493, (2006).

7. M. Tariq, Fiber-Optics and Photonics Technology Conference, IEEE, pp. 21-21, (2007).

8. X.Zhu, and J.M Kahn, IEEE Transactions on Communication, 50, 8, pp.1293-1300, (2002).

9. J. Li, and M. Uysal, Global Telecommunications Conference, 5, pp. 2654- 2658, (2003).

10. X. Guoliang, Z. Xuping, W. Junwei, and F. Xiaoyong, Proc. SPIE, Optical Transmission, Switching, and Subsystems, 5281, pp. 816-823, (2004).

11. L. C. Andrews, R. L. Phillips, and C. Y. Hopen, Laser Beam Scintillation with Applications, SPIE Optical Engineering Press,Bellingham, Washington, (2001). 